\title{
Myxome odontogène du maxillaire : à propos d'un cas
}

\section{Odontogenic myxoma of the maxilla: report of a case}

\section{MOTS-CLEFS :}

- Myxome odontogène, maxillaire,

tumeur odontogène.

\section{KEYWORDS:}

- Odontogenic myxoma, maxilla,

odontogenic tumor.
AOS 2013;266:28-31

DOI: $10.1051 / \mathrm{aos} / 2013604$

(C) EDP Sciences 2013

\section{Résumé}

Introduction. Le myxome odontogène est une variété rare des tumeurs bénignes des maxillaires, dont l'origine serait la portion mésenchymateuse du germe dentaire. Les manifestations cliniques et radiologiques sont variables et non spécifiques et peuvent prêter à confusion avec d'autres lésions radioclaires.

Observation. Nous décrivons un cas de myxome odontogène du maxillaire droit très envahissant, observé chez un jeune patient de 16 ans. Cliniquement, l'adolescent présentait une tuméfaction maxillaire droite, indolore et ferme à la palpation. Une TDM du massif facial montrait un processus expansif ostéolytique soufflant le maxillaire droit. Une biopsie a été en faveur d'un myxome odontogène.

Discussion. Le diagnostic s'est basé sur des arguments cliniques, radiologiques et anatomopathologiques. L'agressivité locale du myxome odontogène et son taux élevé de récidive imposent un traitement radical au-delà des limites de la lésion. La perte de substance qui en résulte implique une réparation chirurgicale ou prothétique.

\author{
Abstract
}

Introduction. Odontogenic myoma (OM) is a rare and locally invasive benign neoplasm found exclusively in the jaws, derived from embryonic mesenchymal elements of dental anlage. Symptoms and radiographic features are variable, so they can lead to confusion with others radiolucent lesions.

Observation. This article presents a rare case of OM occuring in the maxilla of a 16 year's old male patient. The lesion grows without symptoms and presents as a painlaiss sweeling. Computed tomographics images show osteolytic expansile lesion of the right maxilla invading the maxillary sinus. Histopathological examination of the biopsy specimen revealed a myxoma.

Discussion. Diagnosis is based on clinical, radiological and histological features. The odontogenic myxoma local aggressiveness and high recurrence rate requires a radical treatment beyond the limits of the lesion. Loss resulting substance involves surgical repair or prosthetic.

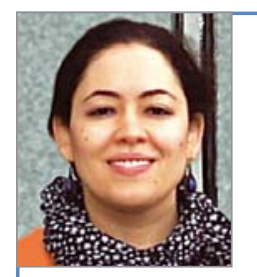

- Dounia KAMAL, Professeur assistante en stomatologie et chirurgie maxillo-faciale, service d'ORL, CHU Hassan II, Fès.

Ayat OUFKIR, Professeur assistante de chirurgie plastique, service d'ORL, CHU Hassan II, Fès.

Hind EL FATEMI, Professeur assistante en anatomopathologie, service d'anatomopathologie, CHU Hassan II, Fès.

Afaf AMARTI, Professeur d'anatomopathologie, et chef du service d'anatomopathologie, CHU Hassan II, Fès.

Mohamed Nourreddine EL ALAMI, Professeur d'ORL et chef du service d'ORL, CHU Hassan II, Fès. 


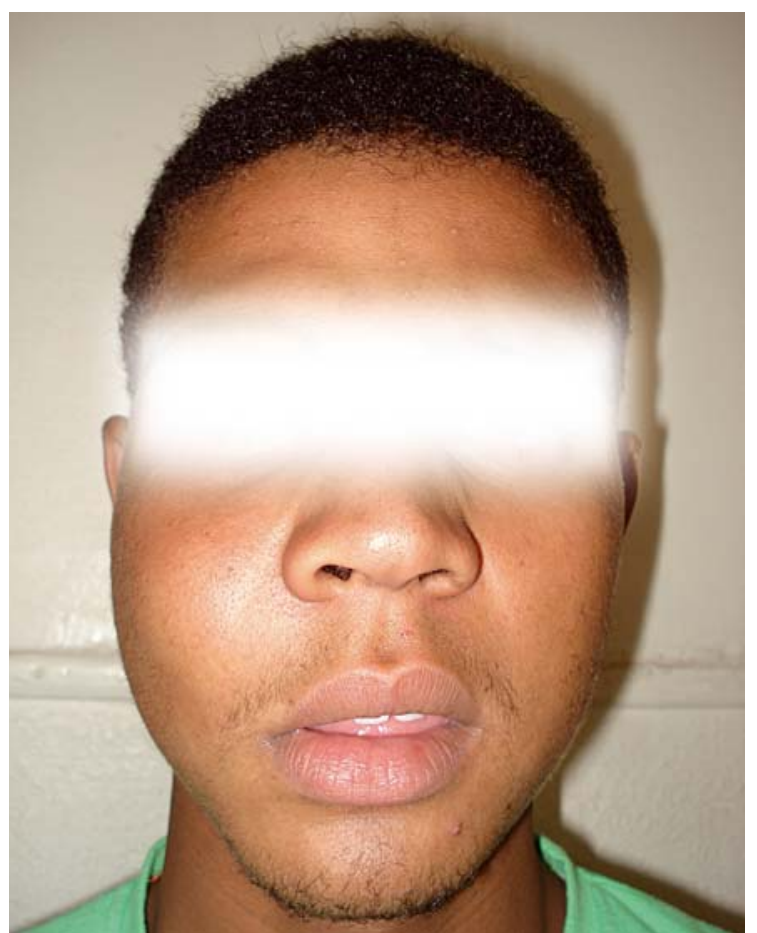

$\triangle$ Fig. 1 :

Vue exobuccale montrant une tuméfaction jugale droite.

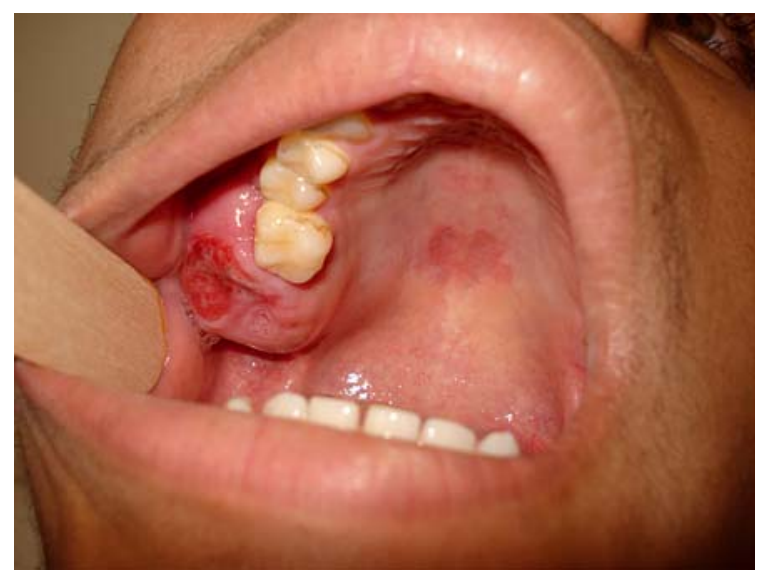

$\triangle$ Fig. 2 :

Vue endobuccale montrant la tuméfaction du secteur molaire supérieur droit avec une ulcération correspondant à la zone de la biopsie.

\section{INTRODUCTION}

Les myxomes des maxillaires sont des tumeurs odontogènes bénignes dorigine mésodermique à fort potentiel d'infiltration et de destruction. Ils surviennent le plus souvent chez l'adulte jeune et ne représentent que $3 \%$ des tumeurs odontogènes et $0,41 \%$ des tumeurs osseuses [1].

Les manifestations cliniques et radiologiques sont variables et non spécifiques et prêtent souvent à confusion avec d'autres lésions bénignes et malignes.

Nous rapportons un cas de myxome du maxillaire avec une revue de la littérature permettant une analyse des difficultés diagnostiques et thérapeutiques.

\section{OBSERVATION}

Un patient âgé de 17 ans, sans antécédents particuliers, a consulté pour une tuméfaction jugale droite évoluant depuis six mois, sans signes oculaires ni neurologiques avec une obstruction nasale homolatérale partielle. L'examen clinique exobuccal (fig. 1) a retrouvé une asymétrie faciale en rapport avec une masse jugale droite indolore, fixe par rapport au plan profond, avec une peau saine en regard. L'examen endobuccal (fig. 2) a retrouvé une tuméfaction du bord alvéolaire du secteur molaire supérieur droit englobant la tubérosité maxillaire droite, la muqueuse de recouvrement était saine avec une mobilité anormale de la 16. L'examen des aires ganglionnaires n’a pas retrouvé d’adénopathies. Le scanner du massif facial a révélé une image radioclaire uniloculaire ostéolytique soufflant les parois du sinus maxillaire droit étendu à la fosse nasale homolatérale et à la paroi médiale de lorbite droite et présentant en périphérie de multiples opacités (fig. 3 et 4).

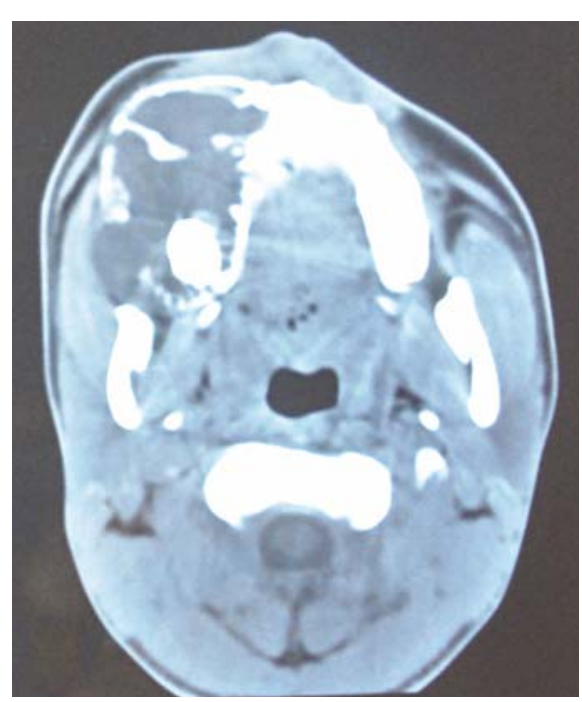

$\triangleleft$ Fig. 3 :

Coupe axiale montrant une lésion lytique maxillaire droite.
Fig. 4 : Coupe axiale montran une lésion lytique maxillaire droite étendue à la fosse nasale homolatérale et à la paroi médiale de l'orbite.

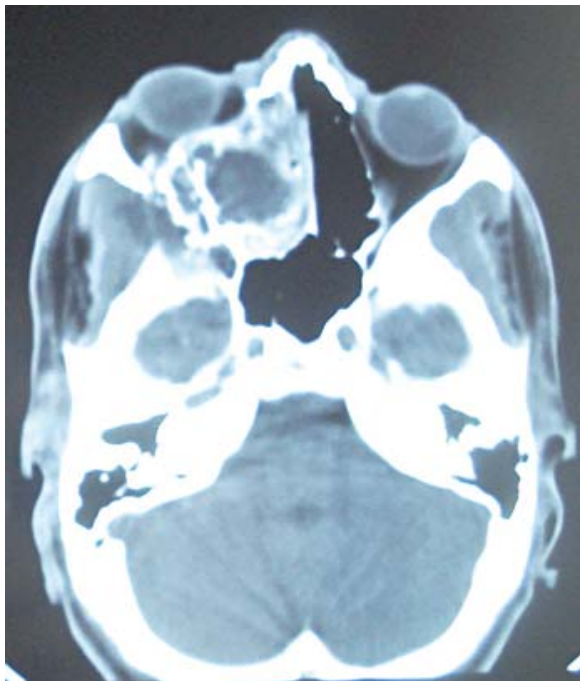


Une biopsie par voie endobuccale était en faveur d'un myxome odontogène. Une exérèse chirurgicale a été réalisée sous anesthésie générale par voie d’abord paralatéronasale, emportant le maxillaire droit par des ostéotomies passant par l'os zygomatique en dehors, la branche montante du maxillaire en dedans, le rebord infra-orbitaire en haut et le secteur molaire en bas (fig. 5 et 6). La paroi postérieure du sinus maxillaire est emportée en monobloc respectant la fosse infratemporale en arrière et un curetage appuyé en haut a entraîné une résection de la lame papyracée du plancher orbitaire qui a été remplacé par une plaque de vicryl pour sustendre le globe oculaire.

L'examen anatomopathologique de la pièce opératoire a révélé une prolifération mésenchymateuse faite de cellules étoilées disposées sur un fond myxoïde (fig. 7 et 8). Les suites opératoires ont été simples sans récidive à un an (fig. 9). Une prothèse adjointe partielle a compensé les pertes dentaires.

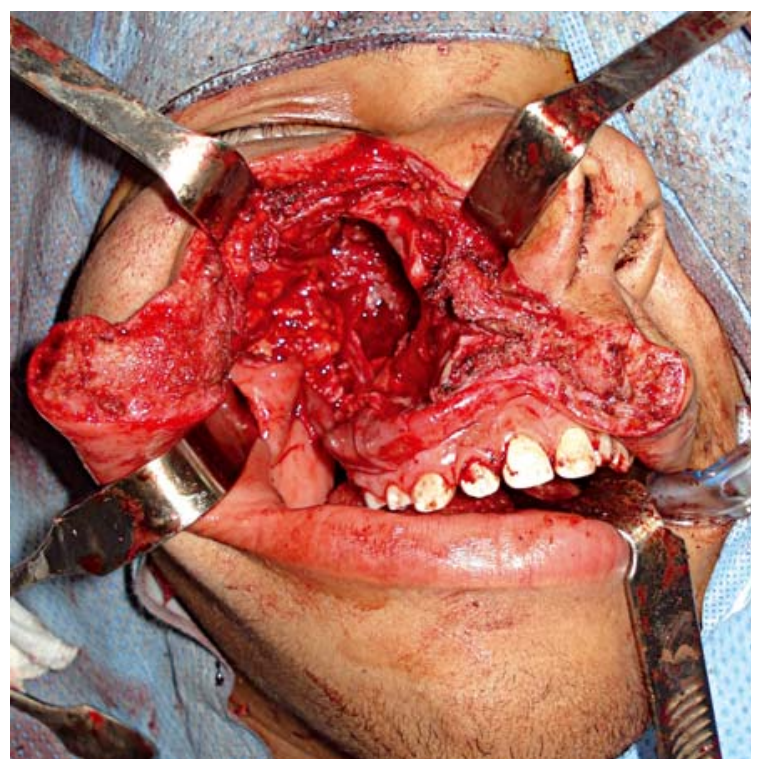

$\triangle$ Fig. 5 :

Vue peropératoire montrant la cavité résiduelle après résection tumorale.

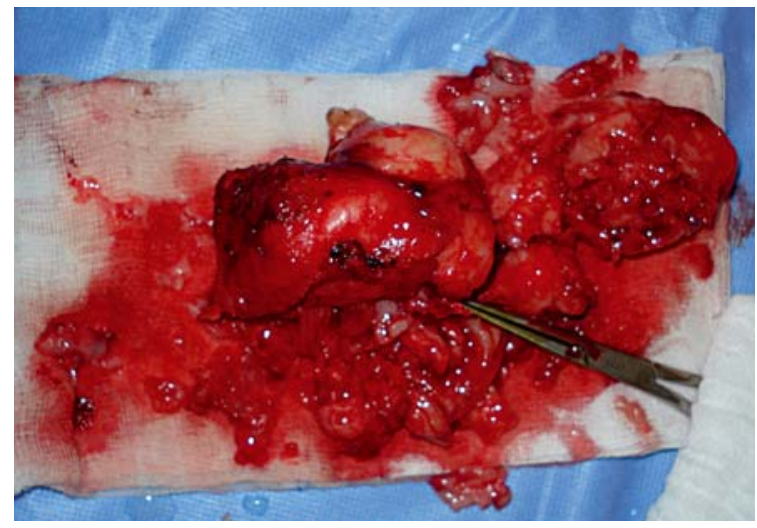

$\triangle$ Fig. 6 :

Photo peropératoire de la pièce opératoire.

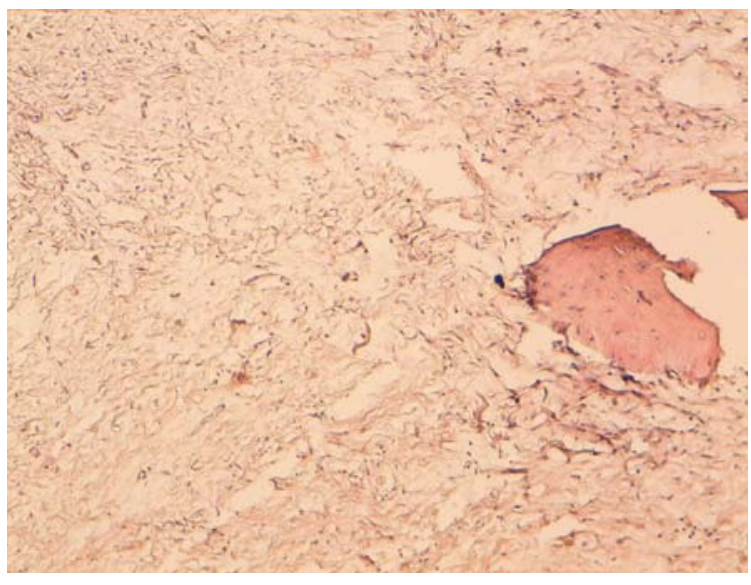

$\triangle$ Fig. 7 :

HES 100 : prolifération myxoïde détruisant l'os.

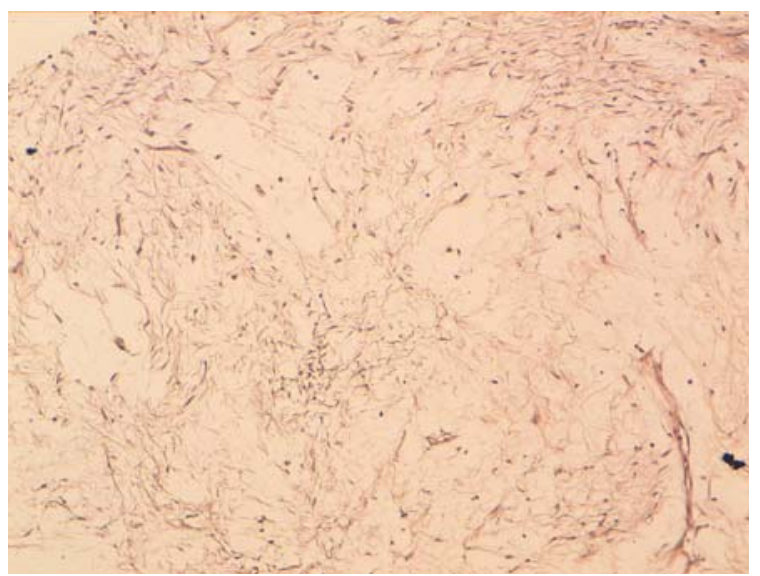

$\triangle$ Fig. 8 :

HES 100 : prolifération paucicellulaire sur fond myxoïde.

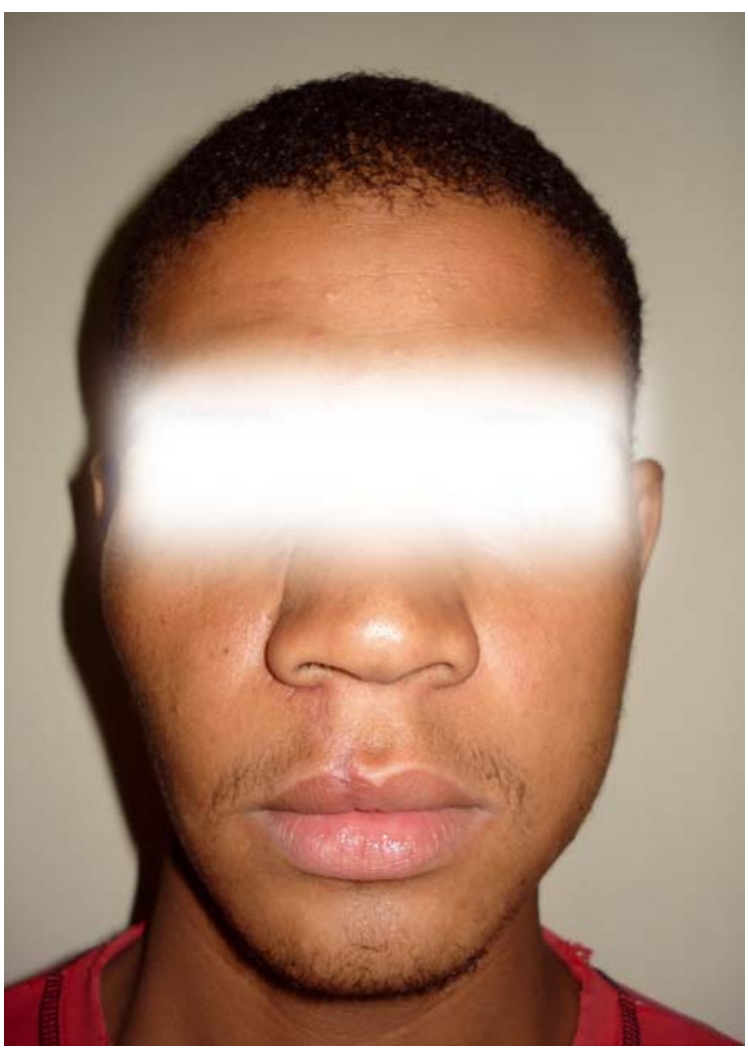

$\triangle$ Fig. 9 :

Photo postopératoire du patient à un an. 


\section{DISCUSSION}

Le myxome odontogène est une tumeur rare qui se développe à partir de la composante mésenchymateuse du germe dentaire, il intéresse la mandibule plus souvent que le maxillaire et rarement les parties molles [2]. Il affecte généralement les adolescents et les jeunes adultes, entre les deuxième et troisième décades de la vie, très rarement les gens avant 10 ans ou après 50 ans [3]. Farman et al. [4] distinguent entre le myxome odontogène maxillaire et mandibulaire, et suggèrent que lâge moyen au moment du diagnostic du myxome odontogène maxillaire chez les hommes est de 29,2 ans pour 35,3 ans chez les femmes, alors que le myxome odontogène mandibulaire chez les hommes survient à un âge moyen de 25,8 ans et chez les femmes à 29,3 ans. Gunhan et al. [5] et Regezi et al. [6] rapportent une incidence plus élevée de ces tumeurs chez les femmes (64-95\%) par rapport aux hommes.

Cliniquement, le myxome a une croissance lente, asymptomatique. Il se manifeste localement par une tuméfaction isolée comme dans notre cas, elle augmente de volume et refoule les corticales, et peut envahir le sinus maxillaire comme dans notre observation. Les symptômes peuvent être des mobilités dentaires, des anomalies dévolution dentaire, parfois des expulsions dentaires au cours de lévolution et un envahissement des tissus mous en cas de destruction des corticales [7] .

Laspect radiologique classique est celui d'une image radio claire, polygéodique. Toutefois, l'aspect d'une lésion kystique monogéodique est possible. Le myxome peut parfois envahir les corticales et les tissus mous, imposant un diagnostic différentiel avec un processus malin. Les images tomodensitométriques ne sont pas spécifiques au myxome, mais doivent être réalisées afin de juger de la taille de la tumeur, de définir ses marges, de diagnostiquer une rupture des corticales et par conséquent dorienter la conduite chirurgicale. Macroscopiquement, le myxome est d'aspect blanchâtre, de consistance molle gélatineuse. Sur le plan histologique, la présence de cellules fusiformes ou étoilées dans un stroma myxoïde confirme le diagnostic [7].

Le diagnostic différentiel de myxome odontogène des maxillaires se pose essentiellement avec l'améloblastome, les sarcomes osseux peuvent être évoqués devant la rapidité dévolution et les aspects lytiques ainsi que la présence de certaines atypies nucléaires dans la composante myxoïde rendant le diagnostic difficile. On peut discuter aussi le chondrome, le fibrome, les tumeurs à cellules géantes et l'hémangiome intra-osseux.

Le traitement est l'exérèse chirurgicale qui peut être : I une énucléation conservatrice pour des lésions de petites tailles non extensives quand lobservance est certaine, tout en sachant que le risque de récidive est deux fois plus élevé pour les localisations maxillaires [8] ; y ou un traitement radical avec une résection osseuse à $1,5 \mathrm{~cm}$ des limites de la lésion entraînant une perte de substance osseuse + /- des parties molles imposant une chirurgie réparatrice immédiate ou différée ou une restauration prothétique.

Une surveillance clinique et radiologique régulière postopératoire prolongée, tous les six mois pendant cinq ans, reste indispensable.

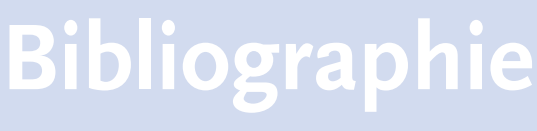

[1] Wong GB. Large odontogenic myxoma of the mandibule treated by sagittal ramus osteotomy and peripheral ostectomy. J Oral Maxfac Surg 1992;50:1221-4.

[2] El H. Bayi, et al. Myxome odontogène des maxillaires. Rev Stomatol Chir Maxillofac. 2006;107(5):389-92.
[3] Shah A et al. Odontogenic myxoma of the maxilla: a report of a rare case and review on histogenetic and diagnostic concepts. Natl J Maxillofac Surg 2011;2:189-95.

[4] Farman, et al. Myxofibroma of the jaws. Br J Oral Surg 1977;15:3-18.

[5] Gunhan O, et al. Odontogenic tumors: A series of 409 cases. Aust Dent J 1990;35:518-22.

[6] Regezi JA, Kerr DA, Courtney RM. Odontogenic tumors: Analysis of 706 cases. J Oral Surg 1978;36:771-8.
[7] Canalis RF, Smith GA, Konrad HR. Myxoma of the head and neck. Arch Otolaryngol 1976;102:300-5.

[8] Heyman O. Nelissen X, Gilon Y, Jacquemin

D. Fissette J. Myxome de la mandibule, à propos d'un cas. Rev Stomatol Chir Maxillofac 2002;103(4):239-41. 